\title{
Determining the visual angle of objects in the visual field: an extended application of eye trackers
}

\author{
Scovil C.Y. ${ }^{1}$, King E.C. ${ }^{1,2}$, Maki B.E. ${ }^{1-5}$
}

Affiliations:
$\begin{aligned} & { }^{1} \text { Centre for Studies in Aging, Sunnybrook Health Sciences Centre; } \\ & { }^{2} \text { Institute of Biomaterials and Biomedical Engineering, } \\ & { }^{3} \text { Institute of Medical Science, and } \\ & { }^{4} \text { Department of Surgery, University of Toronto; } \\ & { }^{5} \text { Toronto Rehabilitation Institute }\end{aligned}$

Number of text pages and figures: 7 pages ( 7 pages max), 2 figures

Correspondence: Brian E. Maki, PhD, PEng

Centre for Studies in Aging, Sunnybrook Health Sciences Centre, 2075 Bayview Avenue, Toronto, Ontario, CANADA M4N 3M5

Tel: (416) 480-6100 ext. 3513; Fax: (416) 480-5856

e-mail: brian.maki@ sri.utoronto.ca

Acknowledgment: Supported by the Canadian Institutes of Health Research (operating grant \#MOP-13355 held by BEM and a CGS-M graduate scholarship held by ECK). ECK also held graduate scholarships from the Toronto Rehabilitation Institute and University of Toronto (Vision Science Research Program, and Institute for Biomaterials and Biomedical Engineering).

Index Terms: eye movements, gaze behavior, central vision, peripheral vision, saccades, visual angles 


\section{ABSTRACT (50 words, max 50 for short communications)}

Many eye-tracker systems display the point of central gaze fixation on video images of the viewed environment. We describe here a method for determining the visual angles of objects

located in the periphery. Such data are needed to study the potential contributions of peripheral vision during cognitive and motor tasks. 


\section{Introduction}

Eye trackers (ET's) are commonly used to study gaze behaviour in a wide range of research fields (e.g. visual-vestibular systems, visual attention, motor control) and activities (e.g. locomotion, sports, driving, reading) [1]. While some ET's simply record eye movements, other ET systems display the point of central gaze fixation on video images of the viewed environment, as recorded by a head-mounted or remote "scene" camera [1]. Such systems allow the object that was fixated to be identified, but provide no quantitative information about the locations of objects in more peripheral regions of the visual field.

The human eye focuses incoming light rays most accurately on the fovea, the retinal area of greatest visual acuity (visual angles $<2.5^{\circ}$ ), and the surrounding macula (visual angles $<9^{\circ}$ ) also provides high acuity [2]. Although acuity decreases as the visual angle of the object moves farther into the periphery [2], studies have demonstrated that peripheral regions of the visual field do provide information that is important in many cognitive and motor tasks (e.g. spatial location [3-5] or detecting the movement of objects [6]). Typically, such findings have been derived from studies in which portions of the visual field are occluded (using special goggles, contact lenses or computer displays $[5,6]$ ) or subjects are instructed to maintain fixation on a specified point $[3,4]$. To study potential contributions of the central and peripheral visual fields under more natural task conditions, it is necessary to be able to determine the visual angles of various objects of interest throughout the duration of the cognitive or motor task being studied.

This communication presents a method for augmenting the point-of-gaze data provided from an ET by plotting associated visual-angle information on the scene-camera image, thus allowing the visual angles of objects of interest in the subject's field of view to be determined. The method presented herein is valid for an ET system for which the spatial relationship between 
the eye and the ET remains constant. This could include a head-mounted ET on a mobile subject, or an environment-mounted ET when the subject's head is immobilized. Example data are presented, and the assumptions and sources of error are discussed.

\section{Methods}

The visual field of the eye may be thought of as a cone, of visual angle $\theta$, centered on the line of gaze (Fig. 1a). As a person views the surroundings, anything that falls within that cone will be visible within the visual angle $\theta$. When using an ET, the three-dimensional scene viewed by the subject is recorded as a two-dimensional video image in the "image plane" of the ET scene camera. The ET calculates the intersection of the line of gaze with the image plane, and plots this "point of gaze", $\mathrm{P}$, on the recorded scene. To determine, with respect to $\mathrm{P}$, the visual angle of objects in the scene, it is necessary to find the coordinates of the "gaze ellipse" created by the intersection of the visual-angle cone and the image plane.

\section{A. Derivation of basic method}

The fundamental objective is to convert distances recorded in the scene-camera image plane to visual angles, $\theta$, given the point of gaze, $\mathrm{P}$, that is provided by the ET software (Fig. 1b). We define the point at which a perpendicular line of gaze would intersect the scene-camera image plane as D. Thus, the distance from any point of gaze to the perpendicular gaze point is PD. If the focal length of the scene-camera lens is OD and gaze is inclined at angle $\alpha$, then:

$$
\alpha=\tan ^{-1}\left(\frac{P D}{O D}\right)
$$

If points $\mathrm{A}$ and $\mathrm{C}$ define the extents of the gaze ellipse, then:

$$
A D=O D \cdot \tan (\alpha+\theta)
$$




$$
C D=O D \cdot \tan (\alpha-\theta)
$$

and the length of the major axis of the ellipse, AC, can be calculated as:

$$
A C=A D-C D
$$

This information then allows the location of the center of the ellipse, B, to be found using:

$$
B D=C D+\frac{A C}{2}
$$

To fully describe the ellipse, the minor axis must also be determined. This is accomplished by finding the "equivalent circle" that results when the visual cone is intersected by a plane perpendicular to its axis, and passing through point B. This plane is represented by points E, B and $\mathrm{F}$ in Fig. 1b. The location of the center of the equivalent circle, point $\mathrm{E}$, is defined by:

$$
P E=B P \cdot \sin \alpha
$$

where $B P=B D-P D$. The radius, $E F$, of the equivalent circle, which defines the semi-minor axis of the gaze ellipse, is then calculated:

$$
E F=O E \cdot \tan \theta
$$

where $O E=O P-P E=\frac{P D}{\sin \alpha}+B P \cdot \sin \alpha$. The gaze ellipse can now be defined in the scenecamera image plane using the coordinates of its center (point B) and the lengths of its semi-major $(\mathrm{AB})$ and semi-minor $(\mathrm{EF})$ axes:

$$
\frac{\left(x-B_{X}\right)^{2}}{A B^{2}}+\frac{\left(y-B_{Y}\right)^{2}}{E F^{2}}=1
$$

The orientation of the ellipse within the scene-camera image plane (i.e. the angle between the ellipse axes and the $\mathrm{x}$ - and $\mathrm{y}$-axes of the camera image) is defined by the orientation of PD. 


\section{B. Calibration procedure}

The above derivation defines the coordinates of the gaze ellipse in "real" units (e.g. mm); however, in order to plot the ellipse on the scene-camera image, it is necessary to express the equations in pixel units, which requires the scene-camera focal length (OD) to also be defined in pixel units. This can be accomplished by performing a simple calibration, which also serves to correct for any lens distortion. The calibration procedure involves fixing the scene camera perpendicular to a known grid (the "calibration plane" shown in Fig. 1), at a known distance $(\mathrm{OD})$, and then digitizing the location of the grid points in the recorded camera image in pixel units. For each grid point $\left(\mathrm{P}^{\prime}\right)$, we know the values of $\mathrm{P}^{\prime} \mathrm{D}^{\prime}$ and $\mathrm{OD}^{\prime}$, and hence the corresponding angle $\alpha$ : $\alpha=\tan ^{-1}\left(\frac{P^{\prime} D^{\prime}}{O D^{\prime}}\right)$. The effective value of OD (in pixel units) for each point $\mathrm{P}^{\prime}$ in the calibration plane (and each corresponding point $\mathrm{P}$ in the digitized image) is then: $O D=P D \cot (\alpha)$, or more directly: $O D=P D\left(\frac{O D^{\prime}}{P^{\prime} D^{\prime}}\right)$, where PD is in pixel units. In allowing OD to vary according to the location of each calibration point, we correct for lens distortion. When analysing actual data, interpolation can be used to estimate the value of OD for any points not explicitly digitized during the calibration.

\section{Translation from scene camera to eye's viewpoint}

The above calculations define visual angles, with respect to the point of gaze, in the scene-camera image plane. Although the ET software correctly locates the point of gaze with respect to the scene-camera image, the scene-camera visual angles that we have calculated will differ from the corresponding visual angles of the eye because the lenses of the eye and the scene camera are not coincident (Fig. 1c). Fortunately, this error can be eliminated by measuring the 
offsets between the eye and the scene camera, and using these to adjust the angular coordinates of the points in the calibration plane.

If the calibration and scene-camera image planes are perpendicular to the subject's perpendicular line of gaze $(\mathrm{OD})$, points $\mathrm{O}_{\text {eye }}$ and $\mathrm{D}_{\text {eye }}$ will be offset from points $\mathrm{O}_{\text {cam }}$ and $\mathrm{D}_{\text {cam }}$ by exactly the same distances in the calibration plane $\left(C_{x}\right.$ and $C_{y}$, Fig. 1c). Thus, the correction can be implemented by shifting point $\mathrm{D}^{\prime}$ in the calibration plane, according to the formulae: $D_{\text {eye }, x}^{\prime}=D_{\text {cam }, x}^{\prime}+C_{x}$ and $D_{\text {eye }, y}^{\prime}=D_{c a m, y}^{\prime}+C_{y}$. In addition, the perpendicular distance between the calibration plane and the observer must be adjusted to reflect the location of the eye rather than that of the camera: $O_{\text {eye }} D^{\prime}=O_{\text {cam }} D^{\prime}+C_{z}$. These changes will then alter the values of $\mathrm{P}^{\prime} \mathrm{D}^{\prime}$ and OD' in the equations presented in Section II-B.

\section{Collection of example experimental data}

Example data were collected, during ambulation, using a head-mounted eye tracker (Model 501, Applied Sciences Laboratories, Bedford, MA, USA) that determines gaze direction by using a miniature camera and infrared light source to record corneal reflections, and plots the point of gaze as a cursor on the image recorded by a miniature head-mounted scene camera. Several other types of ET's are available, including those mounted on glasses or virtual reality goggles, or remote systems where the ET is attached to a surface such as a desk, computer monitor or vehicle dashboard [1]. The equations presented in this paper are applicable to any system in which the head is fixed relative to the scene camera, as noted earlier. Programs to implement the equations derived above were created using Matlab ver. 7.0.1 (Mathworks, Inc., Natick, MA, USA). 


\section{EXAMPLE RESULT}

Figure 2 displays a sample ET scene-camera image, with gaze ellipses plotted to indicate visual angles of $5^{\circ}, 10^{\circ}, 15^{\circ}, 20^{\circ}$ and $30^{\circ}$ with respect to the point of gaze. While the subject's gaze, in this example, was centered on the computer located on the desk, the gaze ellipses indicate the extent to which other objects of potential interest were visible within the central and peripheral visual fields. For example, the phone and the top of the chair were within a $10^{\circ}$ visual angle, and the end of the handrail was within a $15^{\circ}$ visual angle.

\section{DISCUSSION}

The method presented above involves a number of assumptions. These are listed below, along with a discussion of the potential errors resulting from violation of each assumption.

\section{A. Equipment characteristics}

Assumption \#A1: the location of the central gaze cursor, point $P$, is known exactly. All ET's will have some error in calculating the point of gaze, which will be dependent on the design of the ET and the accuracy of the ET calibration. The uncertainty in the gaze location will propagate directly to the gaze-ellipse calculation. For example, an error of $0.5^{\circ}$ in the ET system (a value commonly specified by ET manufacturers) would result in an uncertainty of $\pm 0.5^{\circ}$ in each visual-angle calculation.

Assumption \#A2: the scene-camera lens has no distortion. Most lenses will introduce some distortion. Fortunately, however, this error is effectively eliminated by careful scenecamera calibration, using the procedures described in Section II-B. For our system, which uses an extreme wide-angle ("fish-eye") lens in order to increase the camera's field of view, we determined that failure to implement this correction would have caused errors of up to $30 \%$ in the visual angle (e.g. an error of $\pm 3^{\circ}$ for a visual angle of $10^{\circ}$ ). 


\section{B. Equipment set-up}

Assumption \#B1: the offset distances from the scene camera to the eye are fixed, and known. For our head-mounted system, we could measure these distances with $2 \mathrm{~mm}$ accuracy in each direction. Numerical simulations indicate that this uncertainty results in very small $\left(<0.1^{\circ}\right)$ errors in the visual-angle calculations. Note that other minor violations of this assumption, such as variation in the offset distances [e.g. due to slight $(<1 \mathrm{~cm})$ slippage of a head-mounted ET], would also cause comparatively small errors in visual angle calculations.

Assumption \#B2: the scene-camera image plane is perpendicular to the subject's 'straight ahead' reference line of gaze. The locations of the gaze ellipses are calculated based on the gaze inclination $(\alpha)$ relative to a reference line of gaze (OD) that is assumed to be perpendicular to the image and calibration planes (Fig. 1b). However, the orientation of the camera can be difficult to adjust accurately, particularly in the case of a head-mounted ET system, where the scene camera is very small. The error in gaze-ellipse determination due to error in scene-camera angle increases with both gaze inclination, $\alpha$, and visual angle, $\theta$. For our equipment, scene-camera alignment error was estimated to be $10^{\circ}$, and numerical simulations indicated that this would result in errors of $10 \%$ to $20 \%$ in the visual angle.

Assumption \#B3: the location of the perpendicular gaze point, D (Fig. 1), is known in relation to the image coordinate system. For our setup, we adjusted the scene camera so that the subject's "straight ahead" gaze point was centered in the scene-camera image. We could achieve this centering of point $\mathrm{D}$ to within $5 \%$ of the total image dimensions, which numerical simulations indicated would result in an error of $\pm 1^{\circ}$ in the visual-angle estimates.

In summary, provided that the scene-camera calibration is accurate, violation of assumption \#B2 is likely to have the greatest effect on the gaze-ellipse calculations. However, 
the net effect of all of the assumptions must be considered. In our setup, numerical simulations indicated that with careful calibration, measurement and setup of the ET system, we were able to achieve total errors of $\pm 1.5^{\circ}$ for a visual angle of $5^{\circ}$ and $\pm 2.5^{\circ}$ for a visual angle of $20^{\circ}$. Thus, reasonably accurate estimates of the visual angles of objects within the visual field of the subject are readily achievable using the methods outlined in this paper. We anticipate that this extended application of eye trackers will facilitate future research into the contributions of central and peripheral vision to the control of cognitive and motor tasks and will assist in understanding the visual information that people are able to gather from their surroundings. 


\section{REFERENCES}

[1] M. F. Land, "Eye movements and the control of actions in everyday life," Prog Retin Eye Res, vol. 25, pp. 296-324, May 2006.

[2] H. W. Flynn, S. B. Bressler, G. C. Brown, T. Meredith, and C. D. Regillo, "Section 12: Retina and Vitreous " in Basic and Clinical Science Course, 2003-2004 San Francisco, CA: American Academy of Ophthalmology, 2003, pp. 8-13.

[3] L. Proteau, K. Boivin, S. Linossier, and K. Abahnini, "Exploring the limits of peripheral vision for the control of movement," J Mot Behav, vol. 32, pp. 277-86, Sep 2000.

[4] L. E. Brown, B. A. Halpert, and M. A. Goodale, "Peripheral vision for perception and action," Exp Brain Res, vol. 165, pp. 97-106, Aug 2005.

[5] B. Sivak and C. L. MacKenzie, "Integration of visual information and motor output in reaching and grasping: the contributions of peripheral and central vision,"

Neuropsychologia, vol. 28, pp. 1095-116, 1990.

[6] P. Atchley and G. J. Andersen, "The effect of age, retinal eccentricity, and speed on the detection of optic flow components," Psychol Aging, vol. 13, pp. 297-308, Jun 1998. 


\section{FIGURE CAPTIONS:}

Figure 1: Definition of the optics problem. Panel A illustrates how a given visual angle $\theta$ defines a cone centered about the line of gaze, which is inclined at angle $\alpha$. The intersection of this cone with the scene-camera image plane (i.e. the photosensor of the scene camera) defines the "gaze ellipse". The location of the calibration plane, as defined in Section II-B, is also shown. An exaggerated two-dimensional representation of the above is shown in panel $\mathbf{B}$, for purposes of illustrating the geometric calculations outlined in Equations 1-8. Point $\mathrm{O}$ is centred on the lens of the scene camera, $\mathrm{P}$ is the point of gaze determined by the ET software, and D is the point where the line of gaze would intersect the scene-camera image plane at a right angle. Points A and C denote the outer edges of the "gaze ellipse" (AC is the length of the major axis of the ellipse), $\mathrm{B}$ is the centre of the ellipse, and $\mathrm{E}$ is the centre of the "equivalent circle" (located in a plane perpendicular to the line of gaze), the radius of which (EF) defines the length of the semi-minor axis of the ellipse. Panel $\mathbf{C}$ illustrates (in two dimensions) how the calculations performed in relation to the calibration plane of the scene camera are adjusted to account for the offset between the camera and eye, represented by distances $C_{x}$ (not pictured), $C_{y}$ and $C_{z}$, and thereby correct the calculated visual angles so that they correspond to the visual field of the eye. The correspondence with the eye assumes that there are no significant refractive errors (e.g. due to severe myopia) or that such errors are corrected by lenses worn by the subject.

Figure 2: A sample ET scene camera image showing the point-of-gaze cursor (black square) determined by the ET software and the associated "gaze ellipses" that were determined using the method described in this paper. The plotted ellipses correspond to visual angles of $5^{\circ}, 10^{\circ}, 15^{\circ}$, $20^{\circ}$ and $30^{\circ}$. Whereas the gaze cursor indicates that the subject was looking directly at the computer, the gaze ellipses indicate that visual information about other objects was potentially 
available from other regions of the visual field. For example, the phone and the top of the chair were within a visual angle of $10^{\circ}$ and the end of the handrail was within a visual angle of $15^{\circ}$. 


\section{Figure 1}

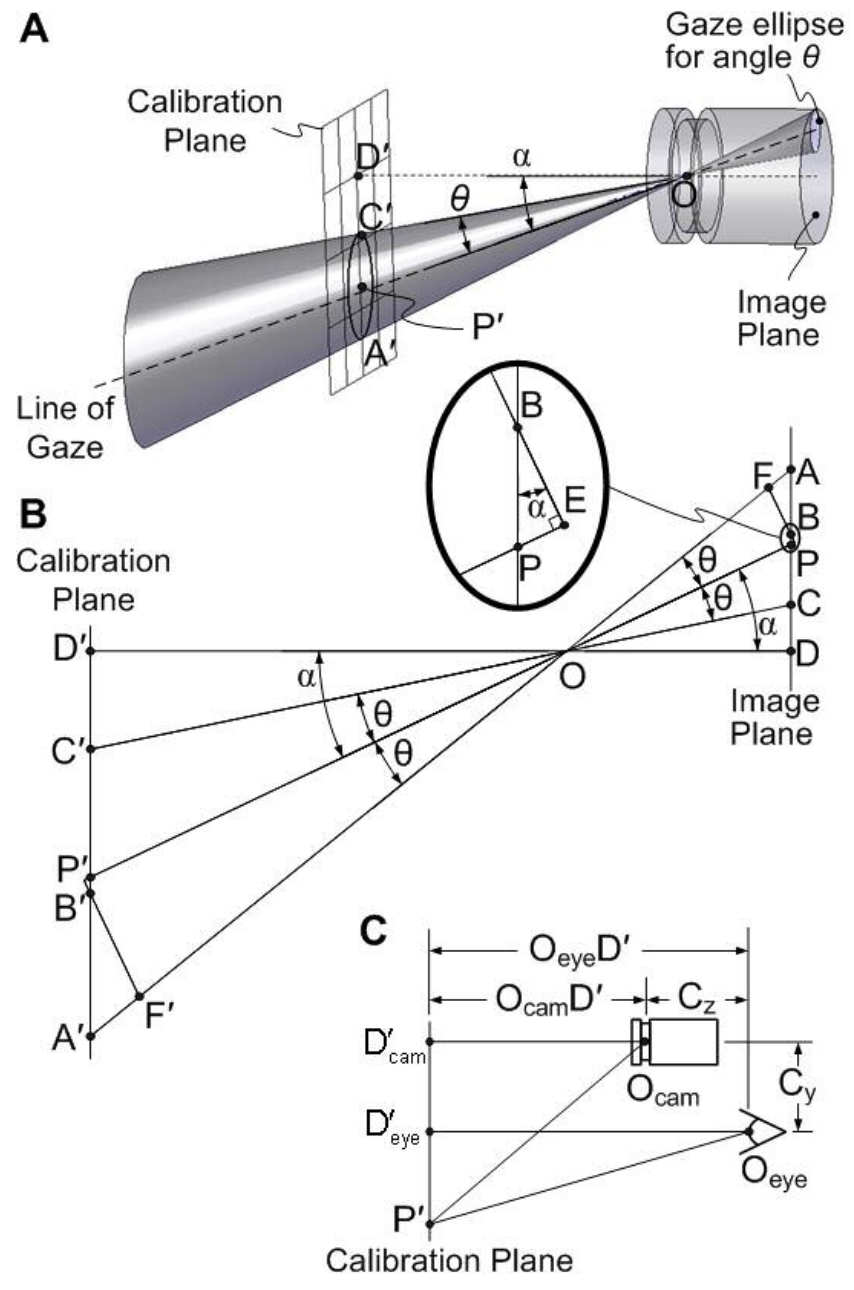


Figure 2

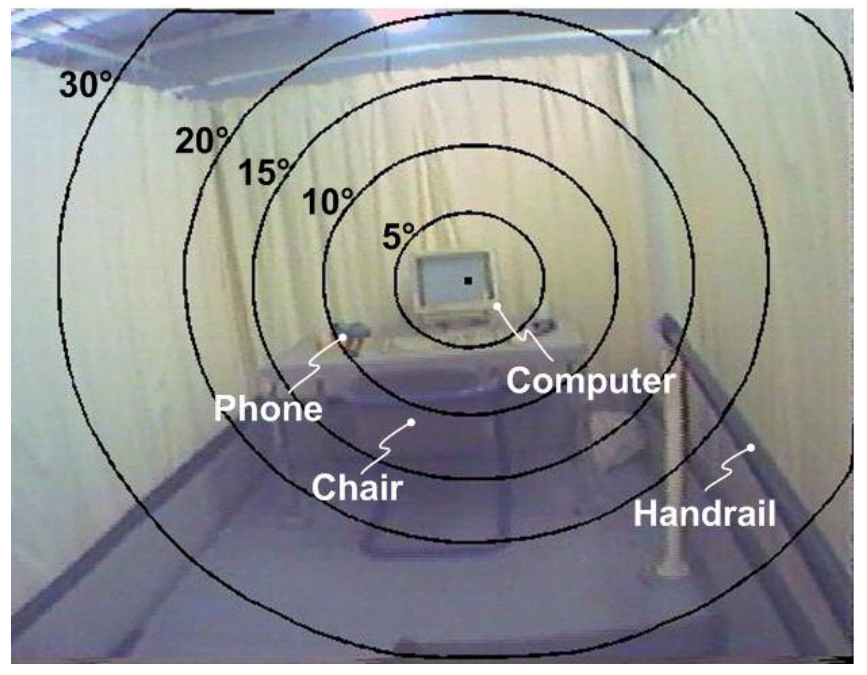

